\title{
Determination of Isotopic Ratio of Boron in Boric Acid Using Laser Mass Spectrometry
}

\author{
Periasamy Manoravi, Mathew JosePh, ${ }^{\dagger}$ Natrajan Sivakumar, and Rajagopalan Balasubramanian \\ Fuel Chemistry Division, Chemistry Group, India Gandhi Centre for Atomic Research, \\ Kalpakkam-603 102, India
}

\begin{abstract}
A reflectron time-of-flight mass spectrometer (RTOFMS) with a laser ablation ion source was used to determine the isotopic ratio of ${ }^{10} \mathrm{~B} /{ }^{11} \mathrm{~B}$ present in boric acid solutions, using graphite as the matrix. The atom $\%{ }^{10} \mathrm{~B}$ values obtained were within $\pm 1 \%$ of the actual ones. The determination of the boron isotopic ratio using this method is, in comparison to other methods, faster, less expensive and easier to perform. The results are compared with the values determined using thermal ionization mass spectrometry.
\end{abstract}

(Received February 4, 2005; Accepted May 9, 2005)

\section{Introduction}

Boron carbide $\left(\mathrm{B}_{4} \mathrm{C}\right)$ is widely used as a control rod material in fast nuclear reactors. The absorption cross-sections of ${ }^{10} \mathrm{~B}$ and ${ }^{11} \mathrm{~B}$ for thermal neutrons are $3837 \mathrm{~b}$ and $5 \mathrm{mb}$, respectively, and for fast neutrons, the cross-section is about $1 \mathrm{~b}$ for ${ }^{10} \mathrm{~B}$ and a much smaller value for ${ }^{11} \mathrm{~B}$. The natural abundances of $\mathrm{B}$ isotopes are: $19.89 \%$ of ${ }^{10} \mathrm{~B}$ and $80.11 \%{ }^{11} \mathrm{~B}^{1}$. Hence, $\mathrm{B}_{4} \mathrm{C}$ pellets enriched in ${ }^{10} \mathrm{~B}$ are used in the fabrication of control rods; the extent of enrichment varies from $60 \%$, for a commercial fast reactor, to as high as $90 \%$ for small core test reactors. Generally, the enriched ${ }^{10} \mathrm{~B}$ is obtained as boric acid using an ion-exchange method. The concentration of boric acid used in the enrichment plant is $\sim 0.1 \mathrm{M}$. It is essential to determine the isotopic enrichment obtained at different stages of plant operation and a large number of samples need to be analyzed. In addition to the above applications, there is also a need to determine the isotopic ratio of $\mathrm{B}$ in heavy water, the moderator used in thermal nuclear reactors. Boric oxide is added to the moderator as a neutron poison during the start-up of a thermal reactor to control the nuclear reactivity.

Thermal ionization mass spectrometry (TIMS) is the conventional method used to determine the isotopic ratio. Though this is the most accurate method, ${ }^{2}$ it is relatively timeconsuming and the boric acid sample has to be converted to sodium borate. ${ }^{2}$ Another method used is based on the use of inductively coupled plasma mass spectrometry (ICP-MS). ${ }^{3}$ This method requires comparatively more expensive instrumentation because of the need for differential pumping and the need to sustain plasma. The laser desorption (vaporization)/ionization source coupled to a time of flight mass spectrometer for mass analysis, provides a fast, less expensive method without the need for extensive sample preparation. Present day solid-state lasers are relatively inexpensive, rugged and compact. ${ }^{4} \mathrm{~A}$ reflectron time-of-flight mass spectrometer (RTOFMS) is easy to construct, operate and maintain. ${ }^{5}$ By the use of time of flight

\footnotetext{
† To whom correspondence should be addressed.

E-mail: mj@igcar.ernet.in
}

measurement, the ion currents due to different masses can all be recorded for each laser pulse. In ICP-MS systems using a quadrupole mass spectrometer, such simultaneous recording of all masses is not possible, and either the value of the mass selected has to be scanned or one must go from one to another. In TIMS systems using magnetic sectors, this is only possible if a multi-collector detector is used.

In this work, we use such a laser mass spectrometry (LMS) method to determine the isotopic ratio of boron in boric acid samples as obtained from the enrichment plants or thermal reactors. The volume of sample needed for analysis is very small $(25-50 \mu \mathrm{L})$. The laser desorption (vaporization) removes only a few picograms of the material from the irradiated part of the surface. This LMS method is simpler than to other similar LMS methods used in the analysis of solid or liquid samples. ${ }^{6}$ In this method, the ions are generated from the sample surface by the use of a single laser pulse without the need for post-ionization. Also, it is not required to accelerate the ions to high energies before analysis ${ }^{7}$ and there is no need to select the energy of the ions other than to reduce the laser power density incident on the sample surface. Hence, the energy spread of the ions have to be less than some value in order to retain good mass resolution.

\section{Experimental}

The details of the LMS experimental facility are reported elsewhere. ${ }^{8}$ Briefly, a Q-switched Nd:YAG laser (M/s Continuum Model NY/61, USA) having a pulse width of $8 \mathrm{~ns}$ and a repetition rate of $10 \mathrm{~Hz}$ was used to transiently heat the boric acid sample. The second harmonic $(532 \mathrm{~nm})$ was used and the transverse mode was mostly $\mathrm{TEM}_{00}$. A home-built reflectron time-of-flight mass spectrometer (RTOFMS), of Mamyrin type ${ }^{5}$ was used for the mass analysis. A quartz lens with a focal length of $25 \mathrm{~cm}$ was used for focusing the laser beam and the target was positioned after the focus. The laser power density incident on the target surface was varied by adjusting the distance between the focusing lens and the target. A micro channel plate (MCP) was used as the detector; the 
signal from the detector was amplified by a fast pre-amplifier (Philips Scientific Model 6950) and fed to digital storage oscilloscope (LeCroy, Model 9350AM).

The measurements on boric acid films were performed using films prepared by placing $\sim 25 \mu \mathrm{L}$ of the sample as a spot on the aluminum disc and then evaporating the solvent under an infrared lamp for $1-2 \mathrm{~min}$. The measurements on the boric acid in copper or graphite matrices were performed using films prepared by thoroughly mixing $\sim 25 \mu \mathrm{L}$ of the sample and a pinch of copper or graphite powder $(\approx$ few $\mu \mathrm{m}$ size) into a slurry on the aluminum disc; this mixture was then dried under an infrared lamp for $1-2 \mathrm{~min}$. The aluminum disc itself was used as the repeller electrode of the ion source. We used only one electrical field for the extraction of the ions. The mass spectrometer was operated in a mode where the field in the ion source defined the ions' flight energy and the values of the retarding and reflecting fields of the reflectron were found from taking the flight time to be independent of the displacement (along the flight axis) of the position of origin of the ion in the source, from the center of the source. The vacuum chamber containing the sample and the mass spectrometer was pumped to a base pressure of $<1 \times 10^{-6}$ Torr by a turbo molecular pump $(1000 \mathrm{l} / \mathrm{s})$. The typical mass resolution of our instrument is about 1000 for gas samples, comparable to any commercial instrument.

TIMS is a well-known method that provides a high degree of accuracy for the determination of isotopic composition of boron. $^{2}$ Hence, for the purpose of comparison of isotopic ratio data, samples studied using the LMS were also studied by TIMS using a VG Micromass 30BK mass spectrometer available in our laboratory.

The samples of boric acid used in this study were from three different sources: namely, natural boric acid, boric acid samples from an enrichment plant, and heavy water containing boric acid.

\section{Results and Discussion}

Figure 1a shows a typical mass spectrum of B obtained for boric acid $(0.1 \mathrm{M})$ having natural abundance. The spectrum was obtained for a boric acid film prepared by placing $\sim 25 \mu \mathrm{L}$ of the sample on the aluminum disc and then evaporating the solvent. The signal is weak. This is because the laser beam is poorly absorbed by the boric acid sample, resulting in transient peak surface temperatures that are not large enough to give rise to a large density of ions on the surface and therefore in the vapor. In order to increase the laser energy absorption, different matrices and substrate were tried. Figure $1 \mathrm{~b}$ shows the mass spectrum for the same sample using a copper strip instead of the aluminum disc where the magnitude of the boron ions' signal is larger. The enhanced peak intensity in the latter case suggests that the substrate surface can absorb the laser beam energy and then transfer it thermally to the film and then to the film surface. One also obtains a larger signal for the boron ions' when the same solution is mixed with fine copper powder or graphite powder and the aluminum disc is used as a sample holder, as shown in Figs. 1c and d. Copper and graphite powders were used because they have larger absorption values for $532 \mathrm{~nm}$ compared to that of aluminum. Being conductive, they also serve to remove any charging effects that will arise if the fluxes of electrons and ions from the surface are different. As can be seen from the figure, the graphite matrix gives the largest (in $\mathrm{mV}$ ) boron ions' signal. Therefore in all subsequent analyses, graphite is used as the matrix.

We also measured the signal intensities (in $\mathrm{mV}$ ) for different
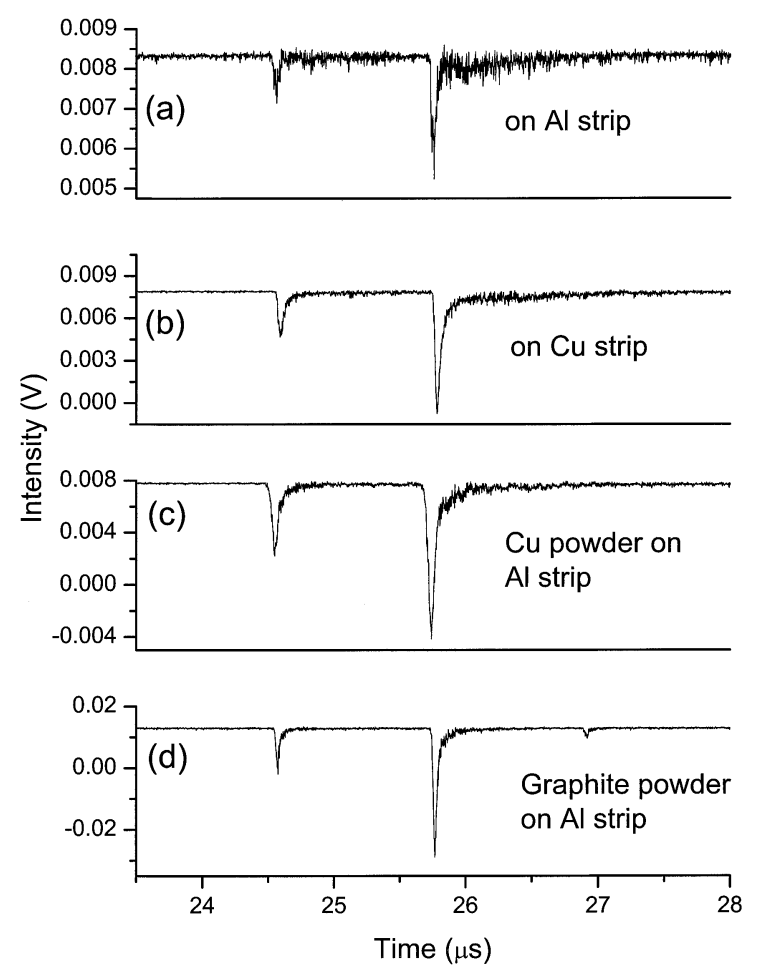

Fig. 1 Typical time-of-flight mass spectrum obtained for boron isotopes from natural boric acid of $0.1 \mathrm{M}$; the sample amount is $25 \mu \mathrm{l}$ sample loaded on (a) aluminum holder, (b) copper holder, (c) copper powder as matrix on aluminum holder, (d) graphite powder as matrix on aluminum substrate. The laser power density is $\approx 7 \times 10^{5} \mathrm{~W} / \mathrm{cm}^{2}$.

boric acid concentrations (using the same volume of the sample solution and mass of the graphite powder). The signal becomes less intense by a factor of 1.7 for $0.1 \mathrm{M}$ and 7 for $0.01 \mathrm{M}$ boric acid compared to that of the intensity obtained with $1 \mathrm{M}$ boric acid. The dependence of signal intensity on the concentration of the sample solution is not linear. This non-linear dependence may arise due to the fact that, during the process of drying, the sample solution inside the slurry volume will continually move to the heated surface through available capillaries and thus the final concentrations on the film surface need not be in the same ratio as those of the original sample solutions.

Typical results obtained for the analysis of $0.1 \mathrm{M}$ boric acid of natural abundance and that of an enriched sample obtained from an enrichment plant are shown in Table 1 along with the values obtained through TIMS measurements. Larger ${ }^{10} \mathrm{~B} /{ }^{11} \mathrm{~B}$ ratios found in the LMS data compared to TIMS results were also observed during the analysis of $\mathrm{B}_{4} \mathrm{C}$ samples, as reported elsewhere. ${ }^{8}$ Results of LMS analyses of boric acid samples having ${ }^{10} \mathrm{~B} /{ }^{11} \mathrm{~B}$ ratios ranging from 0.10 to 2.3 in comparison with results from TIMS analyses are shown as Fig. 2. These results indicate that it is desirable to use a calibration using the TIMS values to improve the accuracy of the data. But the $\pm 1 \%$ accuracy obtained through the LMS measurements in the percentage of ${ }^{10} \mathrm{~B}$ is found to be sufficient for both the enrichment plant and thermal nuclear reactor applications.

Though the sample holder stage used in this study can serve to study only one sample at a time, it is routinely practiced and is easy to accommodate and study several samples on a single holder through adopting a sample holder assembly as done in commercial matrix assisted laser desorption/ionization instruments. The precision of our measurements can be improved by averaging the waveforms for a larger number of 
Table 1 Results of boron isotopic ratio measurements using RTOFMS

\begin{tabular}{|c|c|c|c|c|}
\hline \multirow{2}{*}{$\begin{array}{l}\text { Sample } \\
\text { No. }\end{array}$} & \multicolumn{2}{|c|}{ Natural boric acid } & \multicolumn{2}{|c|}{$\begin{array}{l}\text { Boric acid sample } \\
\text { from enrichment plant }\end{array}$} \\
\hline & ${ }^{10} \mathrm{~B} /{ }^{11} \mathrm{~B}$ ratio & ${ }^{10} \mathrm{~B}, \%$ & ${ }^{10} \mathrm{~B} /{ }^{11} \mathrm{~B}$ ratio & ${ }^{10} \mathrm{~B}, \%$ \\
\hline 1 & 0.258 & 20.51 & 2.564 & 71.94 \\
\hline 2 & 0.265 & 20.93 & 2.432 & 70.86 \\
\hline 3 & 0.250 & 20.02 & 2.585 & 72.10 \\
\hline 4 & 0.246 & 19.77 & 2.489 & 71.34 \\
\hline 5 & 0.266 & 21.01 & 2.449 & 71.01 \\
\hline 6 & 0.290 & 22.45 & 2.351 & 70.16 \\
\hline 7 & 0.279 & 21.79 & 2.358 & 70.22 \\
\hline 8 & 0.278 & 21.73 & 2.522 & 71.60 \\
\hline 9 & 0.279 & 21.80 & 2.366 & 70.29 \\
\hline 10 & 0.255 & 20.32 & 2.454 & 71.05 \\
\hline 11 & 0.280 & 21.86 & 2.340 & 70.06 \\
\hline 12 & 0.257 & 20.44 & 2.433 & 70.87 \\
\hline 13 & 0.242 & 19.48 & 2.493 & 71.37 \\
\hline 14 & 0.243 & 19.55 & 2.433 & 70.87 \\
\hline 15 & 0.268 & 21.15 & 2.268 & 69.40 \\
\hline 16 & 0.270 & 21.26 & 2.467 & 71.16 \\
\hline 17 & 0.253 & 20.18 & 2.582 & 72.08 \\
\hline 18 & 0.275 & 21.55 & 2.401 & 70.60 \\
\hline 19 & 0.279 & 21.79 & 2.449 & 71.01 \\
\hline 20 & 0.279 & 21.81 & 2.500 & 71.43 \\
\hline Mean & 0.257 & 20.97 & 2.447 & 70.97 \\
\hline$\pm \mathrm{SD}$ & \pm 0.014 & \pm 0.88 & \pm 0.084 & \pm 0.71 \\
\hline RSD, \% & 5.3 & 4.2 & 3.4 & 1.0 \\
\hline TIMS & 0.2483 & 19.89 & 2.3602 & 70.239 \\
\hline SD TIMS & \pm 0.002 & \pm 0.163 & \pm 0.01 & 0.36 \\
\hline
\end{tabular}

The serial numbers refer to different measurements on the same sample film: one natural boric acid sample and one enriched in ${ }^{10} \mathrm{~B}$.

laser pulses ${ }^{9}$ than is possible with the DSO used in this study, where a maximum of 1000 sweeps only can be averaged. These measurements suggest that this LMS method, in comparison to other methods that are in use, is faster, simpler and is sufficiently accurate for the purposes discussed above.

\section{Conclusion}

The LMS system developed in our laboratory has been used for the determination of the isotopic ratio of boron present in boric acid, the starting chemical in the production of boron carbide pellets used as control rods in fast nuclear reactors as well as that of the boric acid added to the moderator as neutron poison in thermal nuclear reactors. The measured isotopic data are accurate to within $\pm 1 \%$ in the percentage of ${ }^{10} \mathrm{~B}$ in the boric acid samples. This laser-based technique is better than other mass spectrometric methods that are in use, in terms of cost, ease of construction and ease of operation, and will be useful for routine analyses of a large number of samples as needed for these purposes.

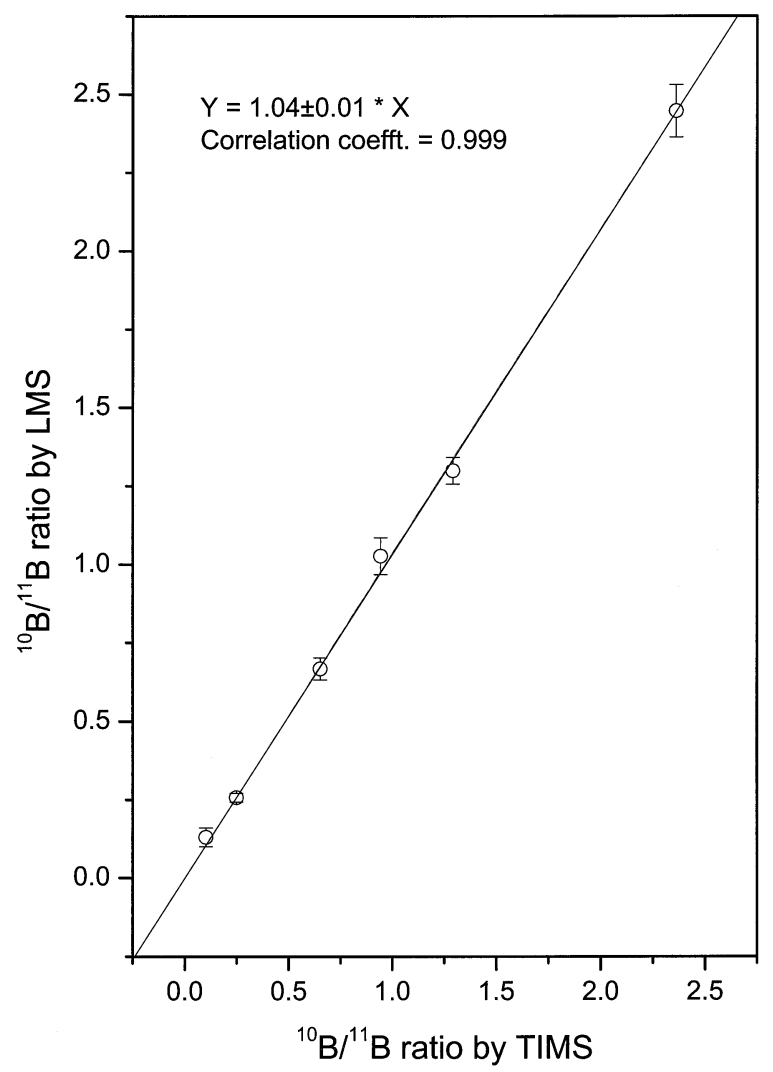

Fig. 2 A calibration plot showing results of ${ }^{10} \mathrm{~B} /{ }^{11} \mathrm{~B}$ ratio obtained by LMS and by TIMS.

\section{References}

1. "Handbook of Chemistry and Physics", 73rd ed., 1992 93, CRC press, 11 - 28.

2. R. N. Sah and P. H. Brown, Biol. Trace Elem. Res., 1998, 66, 39.

3. N. C. Porteous, J. N. Walsh, and K. E. Jarvis, Analyst, 1995, 120, 1397

4. J. J. Zayhowski, J. Alloy Comp., 2000, 303 - 304, 393.

5. B. A. Mamyrin, V. I. Karataev, D. V. Shmikk, and V. A. Zagulin, Sov. Phys. JETP, 1973, 37, 45; N. Sivakumar, M. Joseph, P. Manoravi, P. Parthasarathy, and C. K. Mathews, "Development of Reflectron Time-of-flight Mass Spectrometer", 1996, IGC report, IGC-183.

6. A. A. Sysoev, Eur. J. Mass Spectrom., 2000, 6, 501.

7. J. S. Becker and H. J. Dietze, Spectrosc. Europe, 1998, 10/4, 14 and references therein.

8. M. Joseph, N. Sivakumar, P. Manoravi, and R. Balasubramanian, Rapid Commun. Mass Spectrom., 2004, $18,231$.

9. K. L. Koumenis, M. L. Vestal, A. L. Yergey, S. Abrams, S. N. Deming, and T. W. Hutchens, Anal. Chem., 1995, 67, 4557. 